\title{
Acquisition of tenofovir-susceptible HIV despite consistently high level adherence to daily PrEP as measured by plasma, dried blood spot (DBS) and segmental hair analysis: a case report
}

\author{
Stephanie E. Cohen, MD1, Darpun Sachdev, MD', Sulggi A. Lee, MD², Susan Scheer, $\mathrm{PhD}^{3}$, \\ Oliver Bacon, MD1, Miao-Jung Chen, PhD $^{3}$, Hideaki Okochi, PhD ${ }^{4}$, Peter L. Anderson, \\ PharmD 5 [Full Professor], Mary F. Kearney, $\mathrm{PhD}^{6}$, Susa Coffey, $\mathrm{MD}^{2}$ [Full Professor], \\ Hyman Scott, MD ${ }^{2,7}$, Robert M. Grant, MD ${ }^{8}$ [Full Professor], Diane Havlir, MD² [Full \\ Professor], and Monica Gandhi, MD² [Full Professor] \\ ${ }^{1}$ Disease Prevention and Control Branch, San Francisco Department of Public Health, $3567^{\text {th }}$ \\ Street, San Francisco, CA, 94103 USA \\ ${ }^{2}$ Division of HIV, Infectious Diseases and Global Medicine, University of California San Francisco, \\ 995 Potrero Avenue, Building 80, Ward 84, San Francisco, CA, 94110 USA \\ ${ }^{3}$ HIV Epidemiology Section, San Francisco Department of Public Health, 25 Van Ness Avenue, \\ San Francisco, CA, 94102 USA \\ ${ }^{4}$ Department of Bioengineering and Therapeutic Sciences, Schools of Pharmacy \& Medicine, \\ University of California, San Francisco, 513 Parnassus Avenue, Suite 1113, San Francisco, CA, \\ 94143 USA \\ ${ }^{5}$ University of Colorado Skaggs School of Pharmacy and Pharmaceutical Sciences, Mail Stop \\ C238, 12850 E. Montview Blvd., V20-4101, Aurora, CO, 80045, USA \\ ${ }^{6}$ HIV Dynamics and Replication Program, National Cancer Institute-Frederick, 1050 Boyles Street, \\ Frederick, MD, 21701, USA \\ ${ }^{7}$ Bridge HIV, San Francisco Department of Public Health, 25 Van Ness Avenue, San Francisco, \\ CA, 94102 USA \\ ${ }^{8}$ Department of Medicine, Division of Pulmonary, Critical Care, Allergy, and Sleep Medicine, \\ University of California San Francisco, 2330 Post Street, Suite 420, San Francisco, CA, 94115 \\ USA
}

Corresponding author: Stephanie Cohen, MD, MPH, $3567^{\text {th }}$ Street, San Francisco, CA 94103. Tel: 415-487-5503. Fax: 415-487-5581. stephanie.cohen@ sfdph.org. Alternate corresponding author: Monica Gandhi, MD, MPH, 995 Potrero Avenue $4^{\text {th }}$ floor, San Francisco, CA 94110. Tel: 415-476-4082 × 138. Fax 415-476-6953. Monica.gandhi@ucsf.edu.

Contributors

OB and SC cared for the patient. SEC, DS, SAL, SS, MJC, JO, JWM, PLA, MFK, RG, DH and RMG acquired, analyzed, and interpreted the data. DS oversaw the public health investigation. SS and MJC oversee SFDPH HIV surveillance. SEC, DS and MG drafted the manuscript. All authors critically revised the manuscript.

Publisher's Disclaimer: This is a PDF file of an unedited manuscript that has been accepted for publication. As a service to our customers we are providing this early version of the manuscript. The manuscript will undergo copyediting, typesetting, and review of the resulting proof before it is published in its final citable form. Please note that during the production process errors may be discovered which could affect the content, and all legal disclaimers that apply to the journal pertain.

Declaration of Interests

All other authors declare no competing interests. 


\section{Summary:}

Background: Pre-exposure prophylaxis (PrEP) with emtricitabine/tenofovir (TFV) disoproxil fumarate (FTC/TDF) is highly protective against HIV infection. We report a case of TDFsusceptible, FTC-resistant HIV acquisition despite high adherence to PrEP, confirmed by PrEP drug level testing.

Methods: Adherence to FTC/TDF was assessed by measuring FTC/TFV levels or their metabolites in plasma, dried blood spots (DBS), and hair. Genotypic and phenotypic resistance of the acquired virus was evaluated by standard clinical tests and by single-genome sequencing (SGS) of > 50 proviruses obtained from peripheral blood mononuclear cells (PBMC). HIV partner services (PS) identified the likely transmission partner.

Findings: A 21 year-old Latino man tested positive for HIV (negative HIV antibody, HIV RNA 559 copies per mL) 13 months after PrEP initiation. He reported excellent adherence to daily PrEP. He was linked to care and immediately started antiretroviral treatment (ART), at which point his RNA was 1544 copies per $\mathrm{mL}$ and his HIV Ab test was positive. The HIV genotype revealed M184V, L74V, L100I and K103N mutations in reverse transcriptase (RT); the phenotype showed susceptibility to TDF and resistance to FTC. FTC/TFV levels by liquid chromatography/tandemmass-spectrometry (LC-MS/MS) measured in 1 centimeter $(\mathrm{cm})$ segments of hair from the scalp indicated consistently high PrEP adherence in each of the preceding six months. TFV-diphosphate and FTC-triphosphate levels in DBS indicated high adherence over the preceding six weeks. TFV and FTC levels measured in plasma ten weeks prior to HIV seroconversion confirmed adherence in the days preceding that visit. The likely transmission partner, named during PS, was not engaged in HIV primary care and the viral genotype was similar.

Interpretation: Acquisition of TDF-susceptible, FTC-resistant HIV infection can occur despite high PrEP adherence. Quarterly HIV and STD screening of patients on PrEP, combined with prompt linkage to care and PS for those diagnosed with HIV, facilitates early diagnosis and prevents further transmission of HIV.

\section{Introduction}

Pre-exposure prophylaxis (PrEP) with combination emtricitabine/tenofovir (TFV) disoproxil fumarate (FTC/TDF) is highly protective against acquisition of HIV infection among menwho-have-sex-with-men (MSM) as shown in both randomized trials ( $\left.\mathrm{PrEx}^{1}\right)$ and open-label studies (PROUD, ${ }^{2}$ iPrEx OLE, ${ }^{3}$ U.S. Demo Project ${ }^{4}$ ). PrEP has been estimated to be approximately $99 \%$ effective among MSM when taken daily and $96 \%$ when taken at least four days/week in previous population-based modeling studies. ${ }^{5}$ Five cases of HIV seroconversion despite high adherence to PrEP determined via laboratory-based measures have been previously reported (Table 1). ${ }^{6-10}$ Adherence to PrEP can be assessed with pharmacologic measures of PrEP drug levels (or their metabolites) in a biomatrix such as plasma, ${ }^{11}$ dried blood spots (DBS) ${ }^{12}$ or hair. ${ }^{13}$ However, determining whether an individual was highly adherent to PrEP at the time of HIV exposure and subsequent acquisition remains a challenge as pharmacologic measures at the time of HIV diagnosis may not reflect adherence around the time of HIV exposure. Plasma drug levels estimate adherence over the prior one to three days, ${ }^{11}$ while $\mathrm{DBS}^{12}$ and hair ${ }^{13}$ measures estimate adherence over more 
prolonged periods of time. TFV-diphosphate (TFV-DP) and FTC-triphosphate (FTC-TP) levels in DBS estimate drug adherence over the preceding six weeks. ${ }^{12}$ Segmental hair analysis, in which a small thatch of hair is consecutively cut into $1-\mathrm{cm}$ segments starting at the root, estimates adherence over preceding sequential months. Each 1-cm segment of hair corresponds to a one-month period, with the segment closest to the root corresponding to the most recent month. ${ }^{7}$

In four of the five prior published cases of HIV acquisition despite high PrEP adherence, the virus demonstrated an M184V mutation in reverse transcriptase (RT) conferring resistance to FTC, ${ }^{6-8,10}$ and three of these viruses also had resistance mutations conferring reduced susceptibility to TDF (K70R or K65R) (Table 1). ${ }^{6-8}$ Determining whether the observed viral resistance is transmitted or acquired (secondary to incompletely suppressive two-drug antiretroviral therapy (ART) between HIV acquisition and PrEP cessation) can be challenging without an identified transmission partner.

Here we describe a case patient who was diagnosed with acute HIV infection 13 months after initiating daily PrEP, despite self-reported and laboratory-confirmed evidence of high PrEP adherence over the period of time spanning exposure. We describe his genotypic and phenotypic resistance as well as adherence measured by drug levels in plasma, DBS and segmental hair analysis. HIV partner services (PS) was used to identify the likely transmission partner, and HIV single-genome sequencing (SGS) revealed a homogeneous proviral population, consistent with recent acquisition of HIV. ${ }^{14}$

\section{Case Report}

A 21 year-old Latino man initiated oral daily FTC/TDF 200mg/300mg as PrEP at a municipal sexually transmitted disease (STD) clinic in San Francisco, CA, USA. At his PrEP initiation visit, he reported having insertive vaginal and anal sex with cis female and trans female partners; he had reported receptive anal sex with a cis-man at a prior clinic visit. He had a history of two prior episodes of urethral gonorrhea (GC) and had symptomatic urethral gonorrhea at the PrEP initiation visit. He denied symptoms concerning for acute HIV infection. Other than urethral discharge, his exam was unremarkable; he was uncircumcised. He had Medicaid insurance, which covers the cost of PrEP. He was negative for HIV by both a rapid HIV Ab test (Clearview HIV 1/2 Stat-Pak, Alere) and HIV RNA (RealTime HIV-1 quantitative assay, Abbott Diagnostics) via a standard HIV pooling algorithm at the San Francisco Department of Public Health (SFDPH) Laboratory. ${ }^{15}, 16$ Per clinic protocol, he was prescribed 30 days of FTC/TDF as PrEP with two refills. He was instructed to return every three months for a follow-up visit, at which time he would undergo HIV testing by both rapid HIV test and pooled HIV viral load, receive rectal, urethral and pharyngeal STD testing, undergo clinical evaluation and adherence counseling, and be prescribed a three month supply of PrEP.

The patient was HIV-negative at his three month follow-up visit. At the six month visit, he reported being off PrEP for the prior month as he did not realize that he had refills available at the pharmacy. He was HIV-negative at that visit, and was diagnosed and treated for urethral chlamydia and recurrent genital HSV-2. He was counseled about PrEP adherence 
and was prescribed another three month supply of PrEP. At his next visit, which occurred ten months after PrEP initiation, he reported "good" adherence, and his HIV and STD test results were again negative. Three weeks later, he presented to clinic reporting that he had lost his prescription but that he had an adequate supply of pills at home and had not missed any interim doses. A new prescription was called into his pharmacy. At month 12 , he presented with urethral discharge and was diagnosed with gonococcal urethritis by gram stain and was treated. A urine GC nucleic acid amplification test (NAAT) and urethral GC culture were also positive; all other STD testing was negative.

At month 13, he returned for a PrEP follow-up visit. He was asymptomatic. His urethral discharge had resolved. He reported recent methamphetamine use, condomless receptive anal sex with a male partner, and excellent PrEP adherence, with 0 days missed in the past week and one day missed in the past month. His rapid HIV antibody test was negative and his PrEP prescription was renewed. Urine GC NAAT was positive and he was re-treated for gonococcal infection (Figure 2).

Five days after this visit, his HIV RNA result returned at 559 copies per $\mathrm{mL}$. The patient was promptly notified of the result, linked to HIV care and initiated on once daily ART (on day 6 following diagnosis) with FTC/tenofovir alafenamide (TAF) 200mg/25mg, dolutegravir 50 $\mathrm{mg}$, darunavir $800 \mathrm{mg}$ and ritonavir $100 \mathrm{mg}$. When asked about his adherence to PrEP, he said "I took my medications religiously. If I missed a dose I took two pills the next day." On the day of ART initiation, the patient's HIV viral load was 1544 copies per ml; the HIV $\mathrm{Ag} / \mathrm{Ab}$ test (Abbott Architect HIV Ag/Ab Combo Assay, Abbott Diagnostics) was positive, and a confirmatory HIV 1/2 differentiation antibody test (Geenius, Bio-Rad) was positive for bands p24 and gp41 (bands for gp160 and p31 were absent) (Figure 2). HIV genotyping and phenotyping were conducted by Monogram Biosciences ${ }^{\circledR}$ on virus obtained from plasma collected on the day of his first positive HIV RNA (Phenosense GT ${ }^{\mathrm{TM}}$ ) and the treatment initiation visit (GenoSure $\left.\mathrm{MG}^{\circledR}\right)$.

Per standard health department protocols, a Disease Investigative Specialist (DIS) contacted the patient to assist with disclosure of HIV results, linkage to care and partner services. Selfreported adherence by the patient was verified by calling his pharmacy for refill records. The patient's HIV genotype was reported to HIV surveillance as per California HIV reporting laws. ${ }^{17}$

\section{Methods}

The patient provided written consent to enroll in IRB-approved studies of PrEP seroconversion and early HIV infection at the University of California, San Francisco (UCSF) (NCT02656511) allowing additional samples to be collected for investigation. Hair samples collected on the day of ART initiation were analyzed at the UCSF Hair Analytical Laboratory (HAL), ${ }^{13}$ while DBS collected two days after ART initiation were analyzed at the University of Colorado Antiviral Pharmacology Laboratory. ${ }^{12}$ Both laboratories use validated liquid chromatography/tandem mass spectrometry (LC-MS/MS) methods for analysis of PrEP or PrEP metabolite drug levels. The patient also consented to have testing performed on plasma that had been stored (per routine protocol at the SFDPH public health 
laboratory) from a prior PrEP follow-up visit ( 12 weeks before the HIV diagnosis visit) for both plasma drug levels and ultra-sensitive plasma HIV viral RNA testing (via integrase single-copy assay (iSCA)). ${ }^{18}$ Plasma was tested for FTC/TFV concentrations via standard LC-MS/MS-based methods in the Johns Hopkins Clinical Laboratory and Biomarkers Core.

${ }^{11}$ Lastly, SGS was performed on proviral DNA obtained from peripheral blood mononuclear cells (PBMCs) collected seven days after combination ART initiation to look for minority resistance mutations and estimate the approximate duration of infection. ${ }^{19}$ The iSCA testing was performed at the University of Pittsburgh. SGS was conducted at the National Cancer Institute HIV Dynamics and Replication Program.

\section{Role of the funding source}

The funders had no role in study design, data collection and analysis, decision to publish or preparation of the manuscript. The corresponding author had full access to all the data in the study and has final responsibility for the decision to submit for publication.

\section{Results}

Concern that the patient may have acquired HIV in the setting of high adherence to PrEP led to investigation of three possible etiologies of a PrEP breakthrough: 1) Exposure to a viral strain with reduced susceptibility to TDF and/or FTC; 2) Inadequate adherence to PrEP that was not self-reported; or 3) Unrecognized infection at the time of PrEP initiation followed by delayed seroconversion.

\section{Evaluation for reduced susceptibility to TDF and/or FTC:}

Genotypic testing (GenoSure ${ }^{\circledR}$ MG) for the HIV strain isolated from this patient showed notable RT mutations of L74V, L100I, M184V, and K103N, none of which are known to confer resistance to TDF, although M184V confers high-level resistance to FTC. The strain's protease (PR) gene harbored no significant resistant mutations. Phenotypic testing (Phenosense GTTM) confirmed susceptibility to TDF $\left(\mathrm{IC}_{50} 0.377\right.$; fold change 0.39$)$ and resistance to FTC. To increase the sensitivity for detection of drug resistance mutations and to characterize the genetic diversity of the viral population, 55 single proviral genomes were sequenced via SGS. SGS detected minor resistance mutations in the PR gene that were not reported in the standard genotype (D30N (3.6\%), G73S (1.8\%) and G48E (5.5\%). SGS was consistent with the standard genotype in detecting RT mutations: specifically L74V, L100I, $\mathrm{M} 184 \mathrm{~V}$, and $\mathrm{K} 103 \mathrm{~N}$ were detected, but K65R and K70E were not. The genetic diversity of the proviral population (excluding APOBEC-3G induced G>A hypermutants) was 0.001, highly suggestive of acute infection and transmission within the previous few weeks (Figure 1). ${ }^{14}$

\section{Comprehensive adherence evaluation:}

TFV/FTC concentrations in hair in 1-cm segments from a cut hair sample 6-cm in length collected on the day of ART initiation were commensurate with consistently high PrEP adherence (as evidenced by TFV levels in each segment $>0.038$ nanograms/milligrams $(\mathrm{ng} / \mathrm{mg})^{13,20}$ ) over the preceding six months (Figure 2 and 3 ). TFV-DP and FTC-TP levels 
in a DBS sample collected two days after ART initiation were $1012 \mathrm{fmol} / \mathrm{punch}$ and 0.266 $\mathrm{fmol} /$ punch, respectively, indicating $\geq$ four doses per week on average over the preceding six weeks. ${ }^{12}$ TFV and FTC levels measured in plasma 12 weeks before diagnosis of HIV seroconversion were $188.2 \mathrm{ng} / \mathrm{mL}$ and $870.5 \mathrm{ng} / \mathrm{mL}$ respectively (far above trough levels as determined in a directly-observed therapy study) confirming recent dosing preceding that visit (Figure 2). ${ }^{11}$

\section{Evaluation for unrecognized infection during PrEP initiation.}

Additional testing of stored plasma from the visit 12 weeks prior to the diagnosis visit revealed no evidence of plasma HIV by iSCA (lower limit of detection $=1$ copy per ml). ${ }^{18}$

\section{Identification and evaluation of likely transmission partner}

Through HIV partner services, the patient named five sexual partners, of whom two were known to be HIV-positive per SFDPH HIV surveillance. One of these known HIV-positive partners had an undetectable HIV viral load in the prior three months, while the other had never achieved viral suppression over the seven years since initial HIV diagnosis. The case patient reported condomless insertive and receptive anal sex with this HIV-positive partner approximately three weeks prior to his HIV diagnosis. Per the SFDPH's HIV Surveillance program, over the prior three years this potential transmission partner had a peak viral load of 652,730 copies per $\mathrm{mL}$ and the most recent HIV RNA level recorded in surveillance (nine months prior to the case patient's seroconversion visit) was 680 copies per $\mathrm{mL}$.

Based on information shared by the patient during partner services, and in accordance with routine health department activities, we identified the likely transmission partner. The partner was contacted by health department navigation staff (without disclosure that he had been named through partner services) to re-link him into HIV care. The partner spoke to his primary care provider and restarted ART. Clinical laboratory testing performed seven days after the partner re-initiated ART revealed an HIV RNA level of 15,000 copies per $\mathrm{mL}$. The partner's HIV genotype (which is mandatorily reported to surveillance) harbored the same RT mutations as the case patient.

The case patient remains highly adherent to ART, returns regularly for care, and is now on a simplified ART regimen of FTC/TAF 200mg/25mg, and dolutegravir $50 \mathrm{mg}$ with a consistently suppressed plasma HIV RNA four months after diagnosis.

\section{Discussion}

We report the sixth case of HIV seroconversion ${ }^{6-10}$ despite high adherence to daily FTC/ TDF-based PrEP as evidenced by both self-report and pharmacologic measures. Moreover, this case represents the third in which the transmitted viral strain was genotypically (and phenotypically) susceptible to TDF. 9,10 Of the six reported cases, five of the acquired viral strains had the M184V mutation in RT (Table 1), leading to high-level resistance to FTC. Most importantly, we report on the first PrEP breakthrough infection for which all of the following testing was performed to comprehensively assess the mechanism of PrEP breakthrough: Long-term pharmacologic biomarkers of adherence, including PrEP metabolite levels in DBS and PrEP drug levels in segments of hair representing exposure 
over the past six months; short-term pharmacologic adherence measure of TFV/FTC levels in plasma assessed during routine PrEP use; genotypic and phenotypic resistance assays in the case patient; reliable estimate of timing of infection given highly sensitive HIV testing performed at PrEP initiation, throughout PrEP use and 12 weeks prior to HIV diagnosis; and (mandatorily-reported) viral sequence data from the likely transmission partner, confirming resistance was transmitted and not acquired on PrEP.

\section{Possible explanations of PrEP breakthrough}

The reason for PrEP breakthrough in this case remains unclear despite a comprehensive analysis of the most likely etiologies of PrEP failure. ${ }^{21}$ Patient self-report and pharmacologic measures over a prolonged period of time, made possible by the novel method of segmental hair analysis, ${ }^{7}$ support high and consistent levels of adherence. Plasma TFV and FTC levels at the visit 12 weeks prior to his HIV diagnosis confirm adequate dosing in the days prior to that visit, TFV-DP and FTC-TP levels in a DBS sample indicate $\geq$ four doses per week adherence over the six weeks prior to HIV diagnosis, and drug levels in the segments of hair in the first, second and third months prior to HIV diagnosis are consistent with 7 days per week adherence (i.e. $>0.038 \mathrm{ng} \mathrm{TFV} / \mathrm{mg}$ of hair). We attribute the minor disagreement between the TFV levels in hair estimates and the TFV-DP levels in DBS estimates to lab variability. Levels of TFV-DP in DBS in this patient were associated with complete protection from HIV acquisition among MSM in the iPrEx OLE study. ${ }^{3}$ However, as both hair and DBS levels represent long-term cumulative adherence, it is possible that a brief period of non-adherence that remained uncaptured, but coincided with HIV exposure, could explain PrEP breakthrough in this case. Finally, although systemic PrEP drug levels were high, we do not yet understand the role of tissue concentrations of TFV, TFV-DP, FTC or FTC-TP in the protective efficacy of PrEP. Although rectal concentrations of TFV-DP are typically high after TDF administration, ${ }^{22}$ it is possible that this patient (who did not undergo tissue biopsies for drug concentrations) had lower than expected rectal or foreskin concentrations of PrEP drugs.

The patient did report being off PrEP for approximately one month just prior to his sixmonth follow-up visit. Drug levels in hair from segments measuring his PrEP adherence approximately six months prior to HIV diagnosis were confirmed as slightly lower than in subsequent months (0.0672 vs. $0.0889-0.101 \mathrm{ng}$ TFV/mg, respectively), but these TFV hair concentrations are still above the concentrations at which seven days per week adherence patterns were observed in a directly-observed study. ${ }^{13}$ This lapse in PrEP use initially raised a question of whether the case patient could have been infected at an earlier date during a period of relatively lower adherence, with delayed seroconversion. PrEP can delay seroconversion and FTC/TDF dual therapy can partially suppress HIV RNA levels. ${ }^{23}$ However, given that the case patient was HIV negative at the ten-month visit (three months prior to HIV diagnosis) by both HIV RNA pool and iSCA, an earlier seroconversion date is extremely unlikely. Moreover, the timing of exposure to his likely transmission partner, along with the lack of genetic diversity of the proviral population, supports HIV acquisition approximately 21-28 days prior to diagnosis when self-report, DBS and hair measures confirm high levels of adherence. 
Another factor that could have contributed to the inefficacy of PrEP in this case is inflammation of the foreskin in the setting of frequent urethral STDs and recurrent HSV-2. Although STDs are associated with HIV acquisition in the absence of PrEP, ${ }^{24,} 25$ multiple studies suggest that PrEP efficacy is not diminished in the setting of an STD. ${ }^{1,2,4}$ Whether circumcision provides protection for MSM having insertive anal sex is unclear ${ }^{26}$ and there was no evidence of association between circumcision status and PrEP efficacy in the iPrEx study. ${ }^{1}$

This is the third reported case of PrEP breakthrough in the setting of FTC resistance and TDF-susceptibility (Table 1). ${ }^{9,} 27$ In a fourth PrEP breakthrough case from Toronto, the patient's virus had genotypic resistance to both TDF and FTC, but was phenotypically susceptible to TDF. ${ }^{8}$ There is also one case of acquisition of TDF-susceptible HIV-1 infection in an MSM taking TDF monotherapy for the treatment of hepatitis B infection. ${ }^{28}$ In the Partners PrEP study, ${ }^{29}$ which studied PrEP among heterosexual serodiscordant couples, there was no statistically significant difference in the efficacy of TDF versus TDF/FTC in preventing HIV acquisition (67\% vs. $75 \%$; $\mathrm{p}=0.23$ ), though there was a trend towards higher efficacy with combination therapy. In macaques, combination therapy with TDF and FTC for PrEP was more effective in preventing SHIV infection than therapy with a single agent. ${ }^{30}$ There have not been any randomized clinical trials examining the efficacy of TDF monotherapy for PrEP among MSM, although a randomized safety study showed no infections during TDF PrEP use and seven infections in the deferred or placebo arms. ${ }^{31}$ The possibility that the patient in this case was infected by minor variants with resistance to TDF is extremely unlikely given the SGS results of 55 single proviral genomes, which provides $95 \%$ confidence for detecting a variant that comprises $5 \%$ of the viral population.

These cases in sum suggest that FTC resistance alone in the transmitted virus can lead to the failure of FTC/TDF-based PrEP. In the U.S., approximately 0.4-1.05\% of persons newly diagnosed with HIV have a virus with a transmitted M184V mutation. ${ }^{32-34}$ In San Francisco from 2014-2016, of 338 genotypes from individuals newly diagnosed with HIV, only three (0.89\%) had a transmitted M184V mutation. The prevalence of people living with HIV who have both a detectable VL and an M184V mutation will vary from jurisdiction to jurisdiction. Among 1943 individuals living with HIV in San Francisco who had at least one sequence reported to HIV surveillance from 2013-2017, 356 (18.3\%) had an M184V mutation detected in at least one genotype, of whom 62 were not virally suppressed based on their most recent viral load (Personal communication, Mia Chen, SFDPH HIV surveillance, July 2, 2018). Thus up to 62/1943 (3\%) of individuals in San Francisco with HIV have both a virus with an M184V mutation and a detectable viral load, and could potentially transmit HIV to a partner on PrEP.

\section{Conclusion:}

These results, and results from other PrEP breakthroughs in the literature, suggest that despite high levels of adherence, HIV acquisition can occur on FTC/TDF-based PrEP in the setting of FTC-resistance (despite TDF-susceptibility). Since transmitted FTC resistance is extremely rare, and becoming rarer still in the face of more effective antiretroviral therapies, this type of breakthrough should remain infrequent, although the rate of transmitted FTC 
resistance varies by region. If an individual is being exposed to a virus with known resistance to FTC, additional HIV prevention strategies, including condoms, may be warranted to prevent HIV acquisition. Other PrEP strategies besides oral FTC/TDF, including long-acting injectables, are under investigation.

Individuals taking PrEP and health care providers should be aware that PrEP failure is very rare, but not impossible, even with consistent adherence; the majority of PrEP breakthroughs occur in the setting of inadequate adherence to or lack of persistence on PrEP. Achieving high levels of adherence and persisting on medication through ongoing periods of risk is challenging, both for those on PrEP and for those living with HIV. Additional tools to support long-term retention and adherence to PrEP and HIV care are needed.

This case report provides a potential testing and surveillance algorithm for further characterizing individuals who acquire HIV on PrEP involving objective adherence metrics, genotypic and phenotypic resistance testing of acquired virus, and partner tracing and resistance testing. The novel use of segmental hair analysis can provide information on PrEP adherence patterns over time. These findings also strongly support the importance of regular HIV screening in Individuals taking PrEP, and if diagnosed with HIV, the importance of immediate linkage to HIV care and partner services.

\section{Acknowledgements}

We thank the patient, the PrEP team and Nurse Practitioners at SF City Clinic and the care team at the Ward 86 Clinic.

We also would like to thank Mark Marzinke, PhD and the JHMI lab for their assistance with plasma drug level testing, Sharon Maartens at Monogram Biosciences, Inc for assistance with clinical genotyping, Jonathan Spindler at NCI who performed the SGS, Joshua Cyktor and John W. Mellors at the University of Pittsburgh who performed the iSCA testing, and Patricia Defechereux from UCSF who coordinates the SeroPrEP study.

SAL has received investigator-initiated research funding from Viiv Healthcare and Gilead Sciences; PA receives contract work and grant support from Gilead, paid to his institution; RMG has served as a site investigator for clinical trials of investigational PrEP agents sponsored by GSK and Gilead Sciences. DH, SEC and OB have been lead or co-investigators on studies for which Gilead has donated study medications.

Funding:

This work was supported by National Institutes of Health: the National Institute of Allergy and Infectious Diseases (MG: 2R01AI098472 and RMG: R01AI118575); the National Cancer Institute and funding from the Office of AIDS Research (MFK), the UCSF/Gladstone Institute of Virology \& Immunology CFAR (P30 AI027763), the National Institute of General Medical Sciences (SAL: K23GM112526); UCSF Clinical and Translational Research Institute Clinical Research Center (UL1 RR024131).

\section{References}

1. Grant RM, Lama JR, Anderson PL, et al. Preexposure chemoprophylaxis for HIV prevention in men who have sex with men. The New England Journal of Medicine 2010; 363(27): 2587-99. [PubMed: 21091279]

2. McCormack S, Dunn DT, Desai M, et al. Pre-exposure prophylaxis to prevent the acquisition of HIV-1 infection (PROUD): effectiveness results from the pilot phase of a pragmatic open-label randomised trial. Lancet 2016; 387(10013): 53-60. [PubMed: 26364263]

3. Grant RM, Anderson PL, McMahan V, et al. Uptake of pre-exposure prophylaxis, sexual practices, and HIV incidence in men and transgender women who have sex with men: a cohort study. Lancet Infect Dis 2014; 14(9): 820-9. [PubMed: 25065857] 
4. Liu AY, Cohen SE, Vittinghoff E, et al. Preexposure Prophylaxis for HIV Infection Integrated With Municipal- and Community-Based Sexual Health Services. JAMA Intern Med 2016; 176(1): 75-84. [PubMed: 26571482]

5. Anderson PL, Glidden DV, Liu A, et al. Emtricitabine-tenofovir concentrations and pre-exposure prophylaxis efficacy in men who have sex with men. Sci Transl Med 2012; 4(151): 151ra25.

6. Markowitz M, Grossman H, Anderson PL, et al. Newly Acquired Infection With MultidrugResistant HIV-1 in a Patient Adherent to Preexposure Prophylaxis. J Acquir Immune Defic Syndr 2017; 76(4): e104-e6. [PubMed: 29076941]

7. Thaden JT, Gandhi M, Okochi H, Hurt CB, McKellar MS. Seroconversion on PrEP: a case report with segmental hair analysis for timed adherence determination. AIDS 2018; 4 19. doi: 10.1097/ QAD.0000000000001825. [Epub ahead of print].

8. Knox DC, Anderson PL, Harrigan PR, Tan DH. Multidrug-Resistant HIV-1 Infection despite Preexposure Prophylaxis. N Engl J Med 2017; 376(5): 501-2.

9. Hoornenborg E, Prins M, Achterbergh RCA, et al. Acquisition of wild-type HIV-1 infection in a patient on pre-exposure prophylaxis with high intracellular concentrations of tenofovir diphosphate: a case report. Lancet HIV 2017; 4(11): e522-e8. [PubMed: 28919303]

10. Colby DJ, Kroon E, Sacdalan C, et al. Acquisition of Multidrug-Resistant Human Immunodeficiency Virus Type 1 Infection in a Patient Taking Preexposure Prophylaxis. Clin Infect Dis 2018.

11. Hendrix CW, Andrade A, Bumpus NN, et al. Dose Frequency Ranging Pharmacokinetic Study of Tenofovir-Emtricitabine After Directly Observed Dosing in Healthy Volunteers to Establish Adherence Benchmarks (HPTN 066). AIDS Res Hum Retroviruses 2016; 32(1): 32-43. [PubMed: 26414912]

12. Castillo-Mancilla JR, Zheng JH, Rower JE, et al. Tenofovir, emtricitabine, and tenofovir diphosphate in dried blood spots for determining recent and cumulative drug exposure. AIDS Res Hum Retroviruses 2013; 29(2): 384-90. [PubMed: 22935078]

13. Liu AY, Yang Q, Huang Y, et al. Strong relationship between oral dose and tenofovir hair levels in a randomized trial: hair as a potential adherence measure for pre-exposure prophylaxis (PrEP). PLoS One 2014; 9(1): e83736. [PubMed: 24421901]

14. Keele BF, Giorgi EE, Salazar-Gonzalez JF, et al. Identification and characterization of transmitted and early founder virus envelopes in primary HIV-1 infection. Proceedings of the National Academy of Sciences of the United States of America 2008; 105(21): 7552-7. [PubMed: 18490657]

15. Christopoulos KA, Zetola NM, Klausner JD, et al. Leveraging a rapid, round-the-clock HIV testing system to screen for acute HIV infection in a large urban public medical center. J Acquir Immune Defic Syndr 2013; 62(2): e30-8. [PubMed: 23117503]

16. Quinn TC, Brookmeyer R, Kline R, et al. Feasibility of pooling sera for HIV-1 viral RNA to diagnose acute primary HIV-1 infection and estimate HIV incidence. AIDS 2000; 14(17): 2751-7. [PubMed: 11125894]

17. California Department of Public Health. https://www.cdph.ca.gov/Programs/CID/DOA/Pages/ OA_case_surveillance_resources.aspx. (accessed August 20, 2018).

18. Cillo AR, Vagratian D, Bedison MA, et al. Improved single-copy assays for quantification of persistent HIV-1 viremia in patients on suppressive antiretroviral therapy. Journal of clinical microbiology 2014; 52(11): 3944-51. [PubMed: 25187636]

19. Palmer S, Kearney M, Maldarelli F, et al. Multiple, linked human immunodeficiency virus type 1 drug resistance mutations in treatment-experienced patients are missed by standard genotype analysis. Journal of clinical microbiology 2005; 43(1): 406-13. [PubMed: 15635002]

20. Gandhi M, Murnane PM, Bacchetti P, et al. Hair levels of preexposure prophylaxis drugs measure adherence and are associated with renal decline among men/transwomen. AIDS 2017; 31(16): 2245-51. [PubMed: 28832411]

21. Dashwood T, Tan DH. PrEParing for the unexpected: mechanisms and management of HIV preexposure prophylaxis failure Future Virology 2018; epub.

22. Patterson KB, Prince HA, Kraft E, et al. Penetration of tenofovir and emtricitabine in mucosal tissues: implications for prevention of HIV-1 transmission. Sci Transl Med 2011; 3(112): 112re4. 
23. de Souza MS, Pinyakorn S, Akapirat S, et al. Initiation of Antiretroviral Therapy During Acute HIV-1 Infection Leads to a High Rate of Nonreactive HIV Serology. Clin Infect Dis 2016; 63(4): 555-61. [PubMed: 27317797]

24. Bernstein KT, Marcus JL, Nieri G, Philip SS, Klausner JD. Rectal gonorrhea and chlamydia reinfection is associated with increased risk of HIV seroconversion. J Acquir Immune Defic Syndr 2010; 53(4): 537-43. [PubMed: 19935075]

25. Pathela P, Braunstein SL, Blank S, Shepard C, Schillinger JA. The high risk of an HIV diagnosis following a diagnosis of syphilis: a population-level analysis of New York City men. Clin Infect Dis 2015; 61(2): 281-7. [PubMed: 25870333]

26. Millett GA, Flores SA, Marks G, Reed JB, Herbst JH. Circumcision status and risk of HIV and sexually transmitted infections among men who have sex with men: a meta-analysis. JAMA 2008; 300(14): 1674-84. [PubMed: 18840841]

27. Colby D, Kroon E, Sacdalan C, et al. Acquisition of multidrug resistant HIV-1 infection in a patient taking preexposure prophylaxis. Clinical Infectious Diseases 2018 (in press).

28. Davies O, Alexander H, Robinson N, et al. Failure of daily tenofovir to prevent HIV transmission or the establishment of a significant viral reservoir despite continued antiretroviral therapy. J Int AIDS Soc 2014; 17(4 Suppl 3): 19731. [PubMed: 25397477]

29. Baeten JM, Donnell D, Ndase P, et al. Antiretroviral prophylaxis for HIV prevention in heterosexual men and women. The New England Journal of Medicine 2012; 367(5): 399-410. [PubMed: 22784037]

30. Garcia-Lerma JG, Otten RA, Qari SH, et al. Prevention of rectal SHIV transmission in macaques by daily or intermittent prophylaxis with emtricitabine and tenofovir. PLoS medicine 2008; 5(2): e28. [PubMed: 18254653]

31. Grohskopf LA, Chillag KL, Gvetadze R, et al. Randomized trial of clinical safety of daily oral tenofovir disoproxil fumarate among HIV-uninfected men who have sex with men in the United States. J Acquir Immune Defic Syndr 2013; 64(1): 79-86. [PubMed: 23466649]

32. Wheeler WH, Ziebell RA, Zabina H, et al. Prevalence of transmitted drug resistance associated mutations and HIV-1 subtypes in new HIV-1 diagnoses, U.S.-2006. AIDS 2010; 24(8): 1203-12. [PubMed: 20395786]

33. Kim D, Ziebell RA, Saduvala N, et al. Trends in Transmitted HIV-1 Antiretroviral Drug Resistant Mutations, 10 US HIV surveillance areas, 2007-2010; 20th Conference on Retroviruses and Opportunistic Infections 3 3-6, 2013 Atlanta, GA Abstract 149.

34. Banez Ocfemia MC, Saduvala N, Oster AM. Transmitted HIV-1 drug resistance among men who have sex with men, 11 US jurisdictions, 2008-2011. 21st Conference on Retroviruses and Opportunistic Infections 3 3-6, 2014 Boston, MA Abstract 579. 


\section{Research in context}

\section{Evidence before this study}

Long-term data on daily pre-exposure prophylaxis (PrEP) from both trials and real-world use have illustrated that breakthrough HIV-1 infections are rare. Five cases of HIV seroconversion despite high adherence to PrEP (as measured by PrEP drug level testing) have been previously reported. In three of the five prior cases, the virus had resistance mutations reflective of reduced susceptibility to TDF.

\section{Added value of this study}

In this report, we describe a case patient who was diagnosed with acute HIV infection 13 months after initiating daily PrEP with tenofovir disoproxil fumarate (TDF)/emtricitabine (FTC). He had high level adherence to PrEP as measured by multiple pharmacologic biomarkers, and was infected with a TDF-susceptible, FTC-resistant virus. This is the first PrEP breakthrough infection for which all of the following testing was performed to comprehensively assess the mechanism of PrEP breakthrough: long-term and short-term pharmacologic biomarkers of adherence; genotypic and phenotypic resistance assays in the case patient; reliable estimate of timing of infection given highly sensitive HIV testing (performed at PrEP initiation, throughout PrEP use and 12 weeks prior to HIV diagnosis); and viral sequence data from the likely transmission partner, confirming resistance was transmitted and not acquired on PrEP.

\section{Implications of all the available evidence}

HIV infection during consistent PrEP use is extremely rare, and PrEP is a highly effective intervention to prevent HIV acquisition among individuals who are at increased risk for HIV. This case report and others highlight the importance of assessing both genotypic resistance and pharmacologic measures of adherence when evaluating the etiology of HIV acquisition during PrEP use. Our findings add to prior reports of PrEP breakthroughs and suggest that FTC resistance alone in the transmitted virus, despite TDF susceptibility, can lead to the failure of FTC/TDF-based PrEP. 


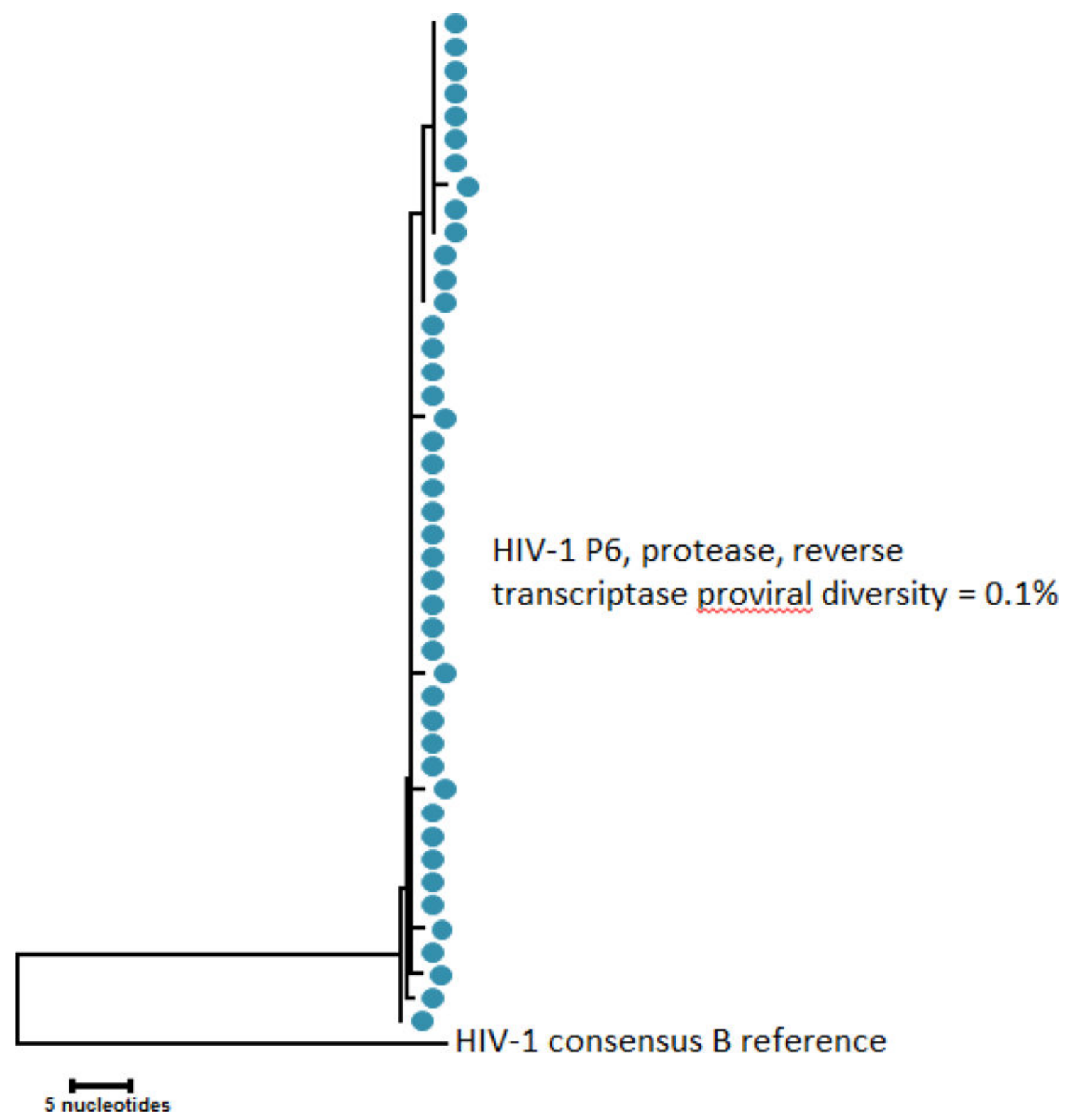

Figure 1. Single-genome sequencing of proviral DNA

Single-genome sequencing was performed on HIV proviral DNA from peripheral blood mononuclear cells obtained seven days after initiation of combination ART. All genomes in the proviral population had L74V, L100I, K103N and M184V RT inhibitor resistance mutations. No genomes had K65R or K70E mutations that would confer resistance to TDF. The genetic diversity of the proviral population was $0.1 \%$, consistent with a very recent transmission event. 


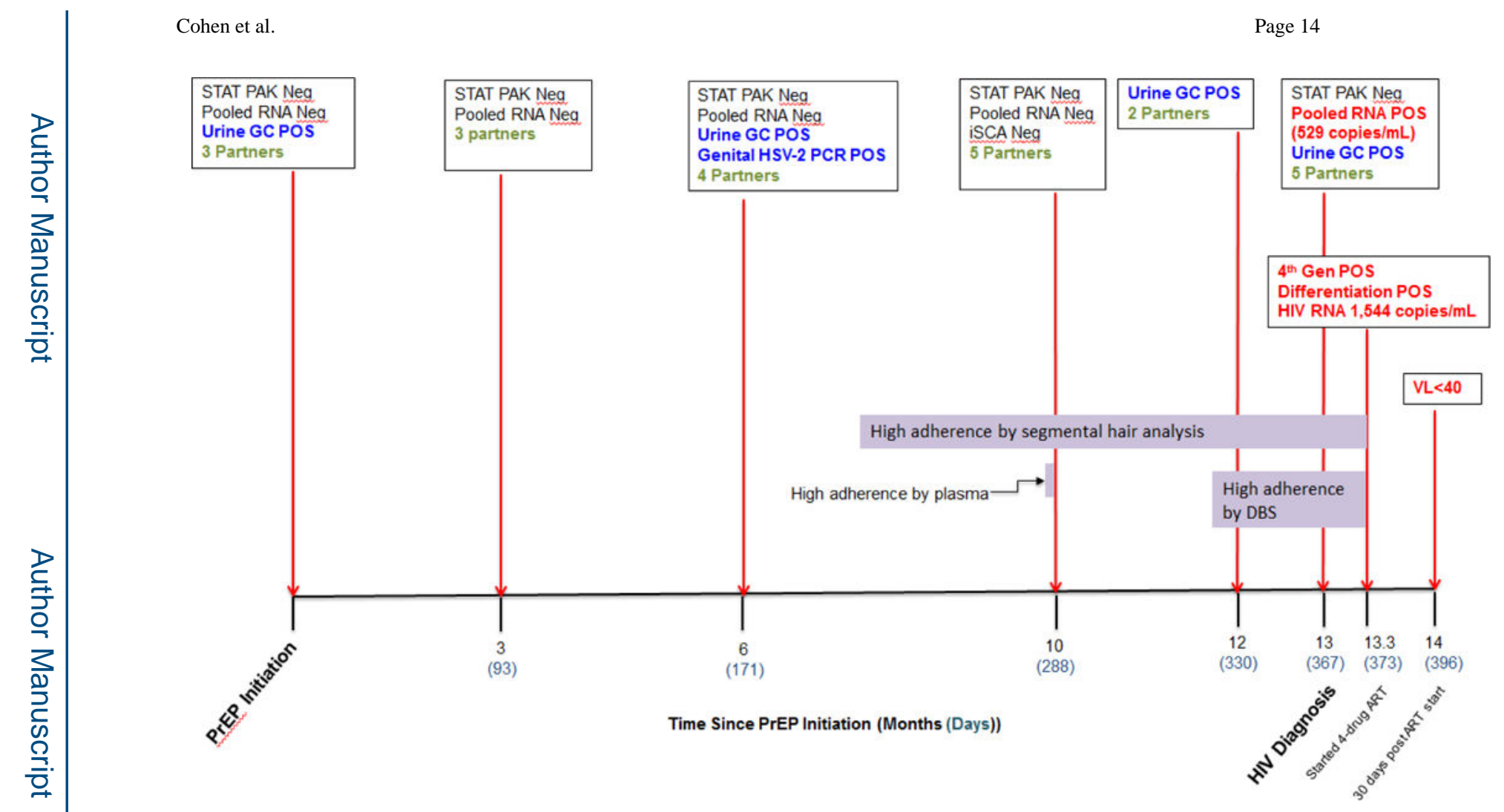

Figure 2. Timeline of events

HIV testing results, positive STD tests and number of sex partners at each visit from PrEP initiation through HIV diagnosis and virologic suppression. Number of sex partners (green) are the self-reported total number of sex partners in the prior three months. Horizontal bars (purple) represent the time period reflected by the specific pharmacologic adherence measure, i.e. plasma levels measure adherence over the prior one to three days, DBS over the prior six weeks and hair over the prior six months. 
Sample

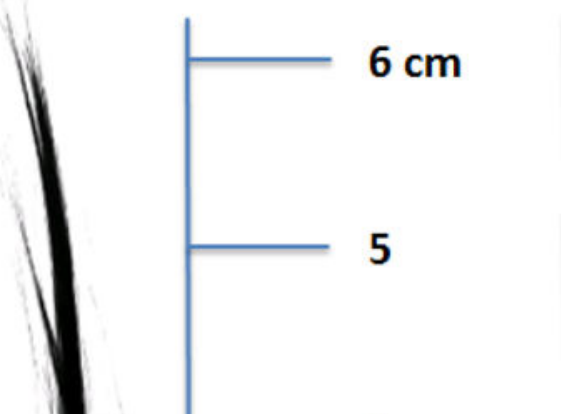

4

2

1

0

\section{Root End}

Figure 3. Segmental hair analysis daily dosing per STRAND study. ${ }^{13}$
Time Span

Month 7-8

Month 8-9

0.0826

Month 9-10

Month 10-11

0.0984

TFV concentration (ng TFV/mg hair)

0.0672

0.0965

Month 11-12

0.0914

Month 12-Diagnosis

TFV concentrations (ng TFV/mg hair) were assessed in the University of California, San Francisco Hair Analytical Laboratory. Hair concentrations > 0.038 ng/mg consistent with 
Table 1.

Summary of published literature on PrEP breakthrough infections with high adherence confirmed by drug level testing

\begin{tabular}{|c|c|c|c|c|c|c|}
\hline Author & Location & $\begin{array}{l}\text { Time } \\
\text { between } \\
\text { PrEP } \\
\text { initiation } \\
\text { and date } \\
\text { of HIV } \\
\text { diagnosis }\end{array}$ & $\begin{array}{l}\text { Major resistance } \\
\text { major mutations } \\
\text { detected on genotype: } \\
\text { NRTI; NNRTI; PI; } \\
\text { INSTI } \\
\text { Phenotype (if } \\
\text { reported) }\end{array}$ & $\begin{array}{l}\text { Resistance } \\
\text { testing } \\
\text { interpretation }\end{array}$ & $\begin{array}{l}\text { Drug level testing* and } \\
\text { interpretation }\end{array}$ & $\begin{array}{l}\text { Interpretation of } \\
\text { likely cause of } \\
\text { PrEP failure } \\
\text { provided by study } \\
\text { authors }\end{array}$ \\
\hline Knox et al. ${ }^{8}$ & Toronto & 24 months & $\begin{array}{l}\text { M41L, D67G, T69D, } \\
\text { K70R, M184V, and } \\
\text { Y215E; Y181C; L10I; } \\
\text { H51Y and E92Q } \\
\text { Phenotype: } \\
\text { FTC resistant; } \\
\text { TDFsensitive }(0.6 \times \\
\left.\text { IC }_{50}\right)\end{array}$ & $\begin{array}{l}\text { FTC: resistant } \\
\text { TDF: low-level } \\
\text { resistant }\end{array}$ & $\begin{array}{l}\text { DBS: } 2297 \mathrm{fmol} / \mathrm{punch} \text { on } \\
\text { day }+24 \text { consistent with } \\
\text { daily dosing in prior } 6 \\
\text { weeks } \\
\text { Plasma: } 152 \mathrm{ng} / \mathrm{ml} \text { on day } \\
0 \\
\text { consistent with recent } \\
\text { dosing }\end{array}$ & $\begin{array}{l}\text { Transmitted drug } \\
\text { resistance to TDF } \\
\text { and FTC }\end{array}$ \\
\hline $\begin{array}{l}\text { Markowitz et } \\
\text { al. }{ }^{6}\end{array}$ & New York & 5 months & $\begin{array}{l}\text { K65R, M184V; } \\
\text { K103S, E138Q, } \\
\text { Y188L }\end{array}$ & $\begin{array}{l}\text { FTC: resistant } \\
\text { TDF: resistant }\end{array}$ & $\begin{array}{l}\text { Hair: } 0.0448 \mathrm{ng} / \mathrm{mg} \text { on day } \\
+35 \\
\text { DBS: } 1478 \mathrm{fmol} / \mathrm{punch} \text { on } \\
\text { day }+35 \\
\text { Hair and DBS consistent } \\
\text { with daily dosing in prior } 6 \\
\text { weeks }\end{array}$ & $\begin{array}{l}\text { Transmitted drug } \\
\text { resistance to TDF } \\
\text { and FTC }\end{array}$ \\
\hline $\begin{array}{l}\text { Hoornenborg } \\
\text { et al. }{ }^{9}\end{array}$ & Amsterdam & 8 months & No major mutations & $\begin{array}{l}\text { FTC: } \\
\text { susceptible } \\
\text { TDF: } \\
\text { susceptible }\end{array}$ & $\begin{array}{l}\text { DBS: } 2234 \mathrm{fmol} / \mathrm{punch} \text { on } \\
\text { day }+6 \text { and } 2258 \mathrm{fmol} / \\
\text { punch } \\
\text { on day }-72 \text {; both } \\
\text { consistent } \\
\text { with daily dosing in prior } 6 \\
\text { weeks }\end{array}$ & $\begin{array}{l}\text { High-inoculum } \\
\text { effect with multiple } \\
\text { exposures; also } \\
\text { with concomitant } \\
\text { LGV infection }\end{array}$ \\
\hline Thaden et al. ${ }^{7}$ & $\begin{array}{l}\text { North } \\
\text { Carolina }\end{array}$ & 14 months & $\begin{array}{l}\text { M184V, K70T, } \\
\text { K65R; K103N }\end{array}$ & $\begin{array}{l}\text { FTC: resistant } \\
\text { TDF: resistant }\end{array}$ & $\begin{array}{l}\text { Hair: } 0.0434-0.0520 \\
\text { ng/mg } \\
\text { on day }+27 \text { consistent with } \\
\text { daily dosing over prior } 3 \\
\text { months } \\
\text { Plasma: } 75 \mathrm{ng} / \mathrm{ml} \text { on day } \\
+27 \\
\text { consistent with recent } \\
\text { dosing }\end{array}$ & $\begin{array}{l}\text { Transmitted drug } \\
\text { resistance to TDF } \\
\text { and FTC or } \\
\text { acquisition of HIV } \\
\text { during period of } \\
\text { lower adherence } \\
\text { with subsequent }\end{array}$ \\
\hline Colby et al. ${ }^{10}$ & Thailand & 8 weeks & $\begin{array}{l}\text { M184V; A98G, } \\
\text { K103N }\end{array}$ & $\begin{array}{l}\text { FTC: resistant } \\
\text { TDF: } \\
\text { susceptible }\end{array}$ & $\begin{array}{l}\text { Hair: } 0.126 \mathrm{ng} / \mathrm{mg} \text { on day } \\
+4 \\
\text { consistent with daily } \\
\text { dosing } \\
\text { in the prior } 6 \text { weeks } \\
\text { Plasma: TDF } 25 \mathrm{ng} / \mathrm{ml} \text { on } \\
\text { day }+2 \text { consistent with } \\
\text { recent } \\
\text { dosing }\end{array}$ & $\begin{array}{l}\text { acquired resistance } \\
\text { Transmitted FTC } \\
\text { resistance or } \\
\text { acquisition of HIV } \\
\text { very proximate to } \\
\text { PrEP initiation }\end{array}$ \\
\hline
\end{tabular}

$4^{\text {th }}$ gen $=$ lab-based $4^{\text {th }}$ generation HIV Ag/Ab test. VL $=$ viral load. NRTI $=$ Nucleoside or nucleotide reverse-transcriptase inhibitor. NNRTI $=$ Non-nucleoside reverse-transcriptase inhibitor. PI = Protease inhibitor. INSTI = Integrase strand-transfer inhibitor. IC50 denotes 50\% inhibitory concentration. DBS = Dried Blood Spot. *Testing on day "x" relative to day of diagnosis, where day $0=$ day of HIV diagnosis. 